\title{
Strengthened health systems are needed to tackle COVID-19 in Africa
}

To the Editor-If one were to ask what the most ideal candidate vaccine against COVID-19 for Africa is, it would undoubtedly be a vaccine that is highly efficient when administered orally, is taken in a single dose, is affordable and is viable at room temperature. The reality is that the vaccines against COVID-19 that have been secured through the COVAX program, currently being received in Africa, do not fall into any of those categories, yet a way must found to distribute them equitably, efficiently and effectively. The COVAX program is a collaboration of Gavi, the Vaccine Alliance, the Coalition for Epidemic Preparedness Innovations and the World Health Organization (WHO). Its aim is to accelerate the development and manufacture of vaccines against COVID-19 and to guarantee fair and equitable access to these vaccines for every country in the world.

In considering the global readiness to carry out this mission, and more so, in evaluating the future of healthcare systems, it is imperative to look back at the lessons learned over the past 12 months.

When the coronavirus SARS-CoV-2 first arrived, it found health systems on the continent in a precarious place. Across Africa, governments grappled with a shortage of basic non-pharmaceutical interventions such as masks, personal protective equipment and oxygen-delivery systems, as well as an inadequate number of beds in intensive care units and a lack of rapid diagnostic testing ${ }^{1}$.

While epidemics such as Ebola have revealed where health systems failed, the COVID-19 pandemic has exposed the glaring inequalities that have existed for too long between countries and regions. Examples include the quality of healthcare services offered in private facilities compared with that of public ones, supply chains that are currently being challenged in their ability to maintain ultra-cold-chain requirements, and the fact that access to health financing remains a challenge, which prevent systems from tackling this disease $^{2}$. Kenya needed an additional 1,500 mechanical ventilators to combat the COVID-19 waves, but the question of personnel to run the machines would remain even if procurement had been done in time.
One of the biggest challenges confronted during the pandemic has been the lack of vaccine-manufacturing capacity on the continent. While biotech and pharmaceutical companies do exist in Africa, their ability to supply the continent is far from sufficient, which leaves countries reliant on global supply systems. African countries will be able to access doses of the AstraZeneca vaccine against COVID-19 from India, by way of Oxford, or from Europe, in the case of the Pfizer vaccine. However, the logistics, costs and resulting complexities and politics relating to access and distribution show that Africa must bypass these challenges by meeting its own needs. Vaccine nationalism became a reality during this pandemic as a symptom of the underlying self-preservation of nations and private firms at the expense of global health.

The role of all governments will be critical in this journey toward strengthened health systems and their contribution to global health. Government-sponsored healthcare coverage such as the Kenyan National Hospital Insurance Fund will need to be enhanced to ensure that no citizen has to choose between health and poverty, and to allow universal health coverage to become a reality ${ }^{3}$. Governments will also need to invest in data and research. Gross expenditure on research and development in Africa is only $0.5 \%$ of the gross domestic product, compared with a global average of $2.2 \%$. Locally funded research and development will enable Africans to find solutions that work effectively on their own populations, which is vital to the development of potential treatments.

Creating a national information health-management system that details the disease burden in Africa, from the number of people with chronic diseases to the statistics on non-communicable diseases, will be invaluable and will allow the respective ministries of health to draft better policy and steer the population toward a healthier future.

The number of healthcare workers is also lagging, due to a lack of human-resource capacity. Silos within the system allow a situation in Kenya whereby recent graduates remain jobless while hospitals buckle under the pressure of operating while understaffed. Streamlining these processes and breaking down walls will allow more-sustainable operation.

The pandemic has also altered health-seeking behavior due to imposed lockdowns and social-distancing measures that prevent patients with chronic diseases from accessing medication, prescriptions and review sessions with medical practitioners. Routine immunization has decreased by as much as $60 \%$ in some countries, despite research highlighting the fact that the deaths prevented by routine childhood immunization outweigh the COVID-19 risk ${ }^{4}$. The drop in routine immunizations will further strain health systems and will also impact the quality and, in some cases, the length of patients' lives. Other indirect effects of the pandemic on healthcare indicators need to be considered and corrected. There is also evidence that gains made in tuberculosis epidemiology over the past decade in Africa are now lost.

Despite these challenges, the resilience and collaboration across borders is encouraging ${ }^{5}$. Ties between medical leads in their respective African countries and the WHO and Africa Centers for Disease Control and Prevention have never been stronger. The flow of information through daily updates and meetings has allowed relevant stakeholders to stay informed on developments such as the emergence of new variants and what kind of clinical outcomes to expect.

The Alliance for Accelerating Excellence in Science in Africa has been involved in these collaborative efforts, working together with the WHO, the Africa Centers for Disease Control and Prevention and various donors and funders from outside the continent to fund research on medicines and vaccines, further demonstrating the power of a cohesive response.

In their 2021 annual letter, Bill and Melinda Gates called for the equitable distribution of vaccines against COVID-19, a pointed attack on the vaccine nationalism sweeping high-income countries. When it comes to the infrastructure and human-resource capability of African countries, the opportunity to close inequality gaps must also be considered. Building a world that is capable of meeting sustainable development goals must involve building stronger health systems. To paraphrase the chief scientist at the WHO, 
Soumya Swaminathan, no life is worth more in one part of the world than in another. The world must continue to honor that.

\section{Moses Alobo ${ }^{凶}$}

Alliance for Accelerating Excellence in Science, Africa-African Academy of Sciences, Nairobi, Kenya.

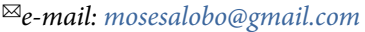

Published online: 10 June 2021

https://doi.org/10.1038/s41591-021-01395-6
References

1. African Academy of Sciences, African Union Development Agency, UK Collaborative on Research Development \& The Global Health Network. The African Academy of Sciences https://www.aasciences.africa/publications/ update-research-and-development-goals-covid-19-africa (2020).

2. African Union, African Centers for Disease Control, World Health Organization \& African Academy of Sciences. World Health Organization Regional Office for Africa https://www.afro. who.int/publications/research-and-development-priorities-covid19-africa (2020).

3. Barasa, E., Ouma, P. O. \& Okiro, E. A. PLoS One 15 e0236308 (2020).

4. Kaja, A. et al. Lancet 8, e1264-e1272 (2020).
5. Sagan, A. et al.. Eurohealth https://apps.who.int/iris/bitstream/ handle/10665/336290/Eurohealth-26-2-20-24-eng.pdf (2020).

\section{Acknowledgements}

D.-F. Ndlovu, communications manager, AAS/AESA, offered support in the writing of this article. M.A. leads the Grand Challenges Africa program at the Alliance for Accelerating Excellence in Science in Africa, a platform of the African Academy of Sciences, African Union Development Agency (AUDA-NEPAD) and global partners.

The author declares no competing interests.

\section{Emergence of measles during the COVID-19 pandemic threatens Pakistan's children and the wider region}

To the editor - In a recent Correspondence by Durrheim et al., the authors described the looming threat of measles amid the COVID-19 pandemic ${ }^{1}$. We agree with the concerns raised by the authors, as shown by the alarming measles outbreak now ongoing in Pakistan, concurrent with the COVID-19 pandemic.

Measles is one of the most contagious viral diseases and is a leading cause of morbidity and mortality, with around 2.6 million annual deaths worldwide. The basic reproduction number of 12 to 18 for the measles virus is the highest of any respiratory virus, and is much higher than that of the coronavirus SARS-CoV-2, the cause of COVID-19 ${ }^{1}$. Despite the availability of a safe and effective vaccine against measles, many countries have experienced measles outbreaks in recent years. In 2019 , there was a precipitous increase in measles outbreaks worldwide, with 413,308 confirmed cases and 207,500 measles-related deaths, compared with 140,000 deaths in $2018,110,000$ deaths in 2017 , and 89,780 deaths in 2016 (refs. ${ }^{1,2}$ ).

Pakistan stands among the top five countries with the largest number of children not vaccinated against measles in 2021, and therefore has the highest toll of measles cases, along with Yemen, Tanzania, India and Nigeria ${ }^{3}$. Measles has been endemic in Pakistan for decades and accounts for $65 \%$ of the total measles burden among 22 countries in the Eastern Mediterranean region ${ }^{4}$.

Since its emergence in December 2019, the COVID-19 pandemic has become a

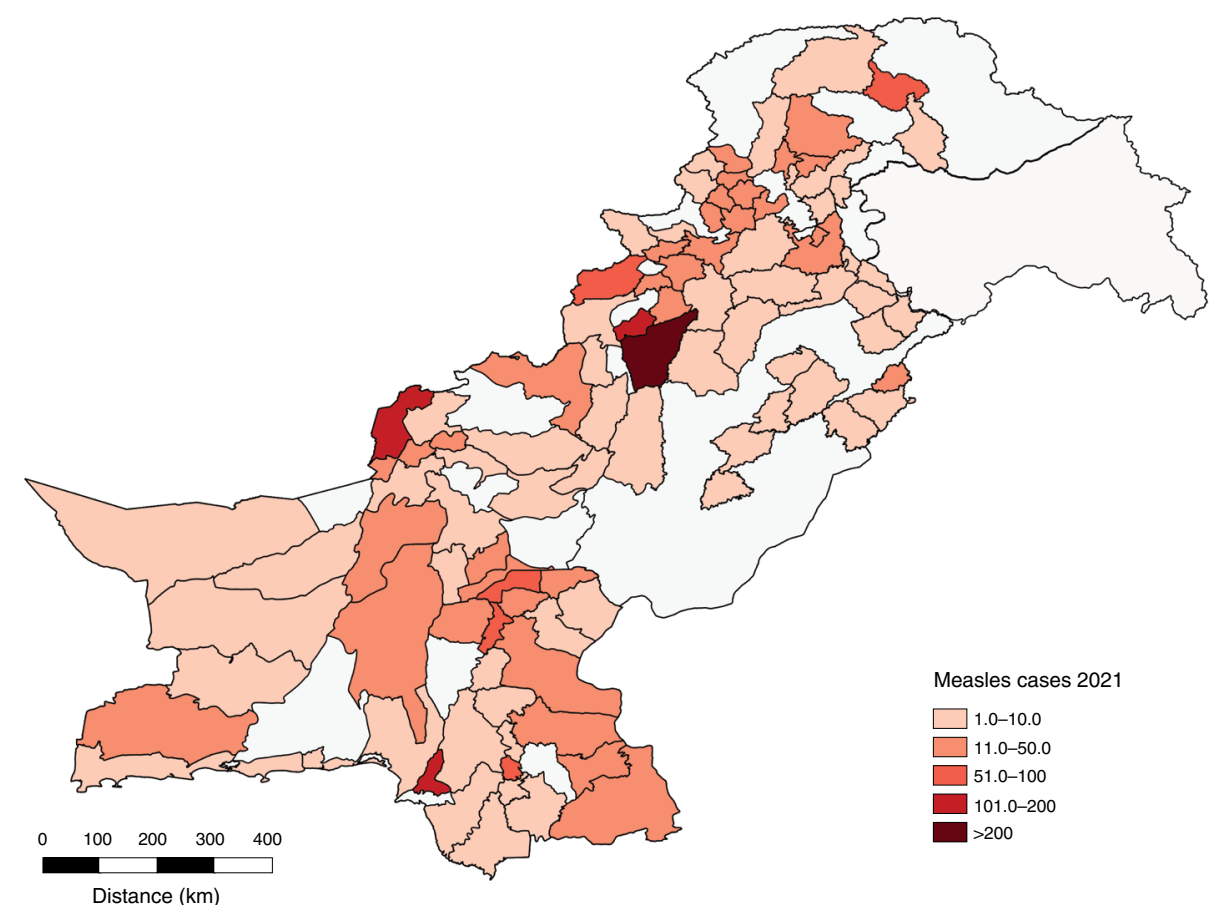

Fig. 1 | The locations of positive measles cases reported in 2021 across Pakistan. Publ. note: Springer Nature is neutral about jurisdictional claims in maps.

worldwide public-health disaster, with more than 172 million confirmed cases and 3.7 million deaths (as of 4 June 2021) reported globally ${ }^{5}$. Likewise, in Pakistan, the toll of confirmed COVID-19 cases reached more than 928,000 , including more than 21,000 deaths, since 26 February 2020 (ref. ${ }^{5}$ ). The third wave of COVID-19 is intensifying in Pakistan and coincides with an unprecedented rise in measles outbreaks across the country, with 79 notified outbreaks, 8,054 confirmed cases and 47 deaths since January 2021 (Fig. 1). 65\% of the total case count comprises children below 5 years of age, $78 \%$ of whom were found to be unvaccinated. 